\title{
ENDOCARCINOMA GÁSTRICO Y EMBARAZO REPORTE DE DOS CASOS Y REVISIÓN DE LA LITERATURA
}

\author{
FORY, JOHANA $A^{1 *}$; ARENAS, VANESA ${ }^{1}$; CARMONA VICENTE ${ }^{2}$; LLANOS JAVIER 3. \\ ${ }^{1}$ Medico Residente Programa Ginecoobstetricia. Hospital Militar Central (Bogotá), Colombia. \\ ${ }^{2}$ Especialista en Ginecología y Obstetricia, Jefe servicio Ginecología \\ Hospital Militar Central (Bogotá), Colombia. \\ ${ }^{3}$ Especialista en Ginecología y Obstetricia, Subespecialista en Medicina Maternofetal. \\ Hospital Militar Central (Bogotá), Colombia. \\ *Correspondencia: johafory@gmail.com,coordinaciongohmc@gmail.com.
}

Transversal 3 \# 49-00 piso servicio ginecología Hospital Militar Central (Bogotā) Colombia. Teléfono: 3486868 extensión 1450

Recibido: 2 Mayo 2017 Aceptado 28 Mayo 2017

\section{RESUMEN}

La incidencia de cáncer durante la gestación es de 1: 1000 a 2000 embarazos, siendo los más frecuentes el de cérvix, mama, ovario, melanoma, tiroides, colon y hematológicos como linfoma y leucemia. A pesar de ser una entidad poco frecuente en el embarazo, se ha visto una tendencia a aumentar en los últimos años debido al incremento progresivo de la edad materna en el momento de la concepción.

El carcinoma gástrico es una patología aún menos frecuente, siendo su incidencia durante la gestación de aproximadamente $0.016 \%$ a $0.026 \%$ en las series de casos de Japón, país con la mayor prevalencia de enfermedad. La cirugía es el tratamiento oncológico menos controversial durante la gestación. En los últimos años, ha cobrado importancia la quimioterapia adyuvante con antineoplásicos de tipo antimetabolito como el 5 fluoracilo (5 - FU), medicamento que ha demostrado mejoría de la supervivencia en pacientes gestantes con adenocarcinoma gástrico. Desafortunadamente, el $\mathbf{9 6 . 7 \%}$ de los casos son diagnosticados en estadíos avanzados, pues su presentación clínica suele confundirse con las manifestaciones propias de la primera mitad de la gestación como son las nauseas y el vomito.

Presentamos dos casos clinicos, el de una gestante de 34 años con embarazo de 19 semanas y 3 días al momento del diagnóstico de un carcinoma gástrico Bormann III, llevada a laparotomía con intención curativa a las 21 semanas con hallazgo intraoperatorio de enfermedad metastásica avanzada y tumor ovárico izquierdo sugestivo de tumor de Krukenberg, a quien se le inicia a las 23 semanas quimioterapia paliativa con esquema FOLFIRI el cual recibió hasta la finalización de la gestación a las 33 semanas por restricción del crecimiento intrauterino y posteriormente durante el puerperio; y el de una gestante de 31 años con embarazo de 26 semanas y 6 días quien se ingresa para estudio de emesis persistente durante la gestación con confirmación diagnóstica de adenocarcinoma gástrico Bormann III asociado a Restricción del crecimiento intrauterino que fue llevada a cesárea a las 34 semanas y en el puerperio a gastrectomía total con vaciamiento ganglionar del area celiaca y reconstrucción en Y de Roux. 
El objetivo de realizar estos reportes surge de la carencia de guias de manejo respecto a dicha entidad durante la gestación debido a la poca frecuencia de presentación de la misma. Es frecuente encontrar discrepancias entre los conceptos en cuanto a conductas médicas se refiere. Disponemos de reportes y series de casos que orientan el manejo hacia un enfoque interdisciplinario dirigido a sopesar los riesgos y beneficios derivados de implementar o no un tratamiento durante la gestación siendo pocos los casos exitosos reportados en la literatura.

Palabras clave: Neoplasias gástricas; Quimioterapia; Teratógenos; Complicaciones neoplásicas del embarazo; Retardo del crecimiento fetal.

\title{
GASTRIC CARCINOMA AND PREGNANCY REPORT OF TWO CLINICAL CASES AND REVIEW OF THE LITERATURE
}

\begin{abstract}
Incidence of cancer during pregnancy is 1 in 1000. The most frequent carcinomas are the cervix, breast, ovaries, melanoma, thyroid, colon and hematological such as lymphoma and leukemia. Although cancer is not frequently associated with pregnancy, there has been a tendency to increase in recent years due to the progressive increase in maternal age at conception.

Gastric carcinoma during pregnancy is a rare pathology, with an incidence of $0.016 \%$ to $0.026 \%$ in the series of cases from Japan, one of the countries with the greatest prevalence of this disease. Surgery is the least controversial type of oncologic treatment during pregnancy. In the last years, the adjuvant chemotherapy with antimetabolite drugs like the 5-fluoracile has gained relevance because it has demonstrated to improve survival in pregnant patients with gastric carcinoma. Unfortunately, the $96.7 \%$ of cases are diagnosed in advanced states because its symptoms can be misinterpreted as pregnancy induced nausea and vomiting characteristic of first half of pregnancy.

We present two Clinical cases. The case of a 34 years old pregnant woman with 19 weeks and 3 days of gestation at the time of diagnosis of a gastric carcinoma in Bormann III stage, she was laparotomized with curative intentions at 21 weeks of gestation with intraoperative findings consistent with advanced metastatic disease and left-sided ovarian tumor suggestive of Krukenberg tumor. At 23 weeks palliative chemotherapy with FOLFIRI was initiated and it was continued until the end of gestation at 33 weeks and later in the puerperium; and the case of a 31 years old pregnant woman with 26 weeks and 6 days of gestation, she was admitted for the study of persistent vomiting during pregnancy with diagnostic confirmation of gastric adenocarcinoma Bormann III associated with intrauterine growth restriction, she was carried cesarean at 34 weeks. In the postpartum she was carried total gastrectomy with lymph node dissection of the celiac area and Roux-Y reconstruction.

The objective of this report arises from the absence of clinical randomized studies about the management of this entity during pregnancy in consequence of the few cases available in the literature. This has been a cause of discrepancies between medical staff regarding therapeutics. There are some available literature series and case reports that guide
\end{abstract}


management to a multidisciplinary approach in pursuit of more benefits and less risk in the context of the pregnant patient, with little cases reported as successful.

Key words: Stomach Neoplasms; Pregnancy Complications; Drug Therapy; Teratogens; Fetal Growth Retardation.

\section{ENDOCARCINOMA GÁSTRICA E GRAVIDEZ RELATÓRIO DE DOIS CASOS E REVISÃO DA LITERATURA}

\section{RESUMO}

A incidência de câncer durante a gravidez é de 1 em 1000. Os carcinomas mais freqüentes são o colo do útero, mama, ovários, melanoma, tireóide, cólon e hematológicos, como linfoma e leucemia. Embora o câncer não seja freqüentemente associado à gravidez, houve tendência de aumento nos últimos anos devido ao aumento progressivo da idade materna na concepção.

O carcinoma gástrico durante a gravidez é uma patologia rara, com incidência de 0,016\% a 0,026\% na série de casos do Japão, um dos países com maior prevalência desta doença. A cirurgia é o tipo menos controverso de tratamento oncológico durante a gravidez. Nos últimos anos, a quimioterapia adjuvante com fármacos antimetabolitos como a 5-fluoracile ganhou relevância porque demonstrou melhorar a sobrevida em gestantes com carcinoma gástrico. Infelizmente, os $96,7 \%$ dos casos são diagnosticados em estados avançados porque seus sintomas podem ser mal interpretados, pois a náusea e os vômitos induzidos pela gravidez são característicos da primeira metade da gravidez.

A presentamos dois casos clínicos. O caso de uma mulher grávida de 34 anos com 19 semanas e 3 dias de gestação no momento do diagnóstico de carcinoma gástrico no estádio Bormann III, foi laparotomizada com intenções curativas às 21 semanas de gestação com achados intraoperatórios consistentes com doença metastática avançada e tumor de ovário esquerdo sugestivo de tumor de Krukenberg. Às 23 semanas, iniciou-se a quimioterapia paliativa com FOLFIRI e continuou até o final da gestação às 33 semanas e mais tarde no puerpério; e o caso de uma mulher grávida de 31 anos com 26 semanas e 6 dias de gestação, foi admitida para o estudo de vômitos persistentes durante a gravidez com confirmação diagnóstica de adenocarcinoma gástrico Bormann III associado à restrição de crescimento intra-uterino, foi realizada cesariana às 34 semanas. No pós-parto foi transportada gastrectomia total com dissecção dos linfonodos da área celíaca e reconstrução Roux-Y.

O objetivo deste relatório decorre da ausência de estudos clínicos randomizados sobre o manejo dessa entidade durante a gravidez em conseqüência dos poucos casos disponiveis na literatura. Esta foi uma causa de discrepâncias entre a equipe médica em relação à terapêutica. Existem algumas séries de literatura disponíveis e relatos de casos que orientam o gerenciamento para uma abordagem multidisciplinar em busca de mais benefícios e menos risco no contexto da paciente grávida, com pequenos casos relatados como bem sucedidos.

Palavras-chave: Neoplasias de estômago; Complicações de gravidez; Terapia de droga; Teratógenos, Retardamento do Crescimento Fetal. 


\section{Introducción}

La incidencia de cáncer en el embarazo se encuentra alrededor de 1 en 1000 (1-3); el cáncer gástrico se presenta en el 0.016 (1) a $0.026 \%$ (2) de los embarazos sin encontrarse dentro de los primeros lugares que son ocupados por el cáncer de seno, con 1 en 3000 casos, seguido por cérvix, linfoma Hodgking y cáncer de ovario (4-6), siendo así una entidad rara en el grupo de gestantes sobre la cual aún no existen estudios aleatorizados controlados con respecto a su abordaje y tratamiento (7).

El $67 \%$ de los embarazos ocurren en mujeres mayores de 30 años, lo que refleja el incremento progresivo de la edad materna al momento de la concepción (8) y con esto la presentación de cáncer es cada vez más frecuente dentro de la población gestante, sin embargo, su diagnóstico suele ser tardío (96.7\% de los casos) (4) por la presentación clínica inespecífica como náuseas, vómito, anorexia, HVDA, plenitud gástrica y epigastralgia, que en muchas ocasiones suele atribuirse a los cambios fisiológicos propios de la primera mitad del embarazo. Cabe destacar, que posiblemente el estímulo hormonal generado en la gestación pueda jugar un papel importante en la exacerbación de muchos cánceres y el aumento de la vascularización favorezca la diseminación tumoral.

Las series de casos más grandes disponibles en la actualidad provienen en su mayor parte de Japón, país con alto registro de cáncer gástrico, destacándose 3 publicaciones: Ueo (1991) con 104 casos, Jaspers (1999) con 92 casos y Maeta (1995) con 14 casos, en las cuales predominaron los carcinomas difusos, pobremente diferenciados diagnosticados generalmente en estadíos avanzados de la enfermedad (5).

El método más sensible para su diagnóstico es la endoscopia de vías digestivas altas. La resonancia magnética nuclear y la ultrasonografía han demostrado ser seguras en el embarazo por tratarse de radiación no ionizante y no generar efectos adversos en el feto (9-11) Si se consideran necesarios los exámenes radiográficos, la exposición total de radiación fetal debe ser "tan bajo como sea razonablemente posible", dado que los efectos inducidos por la radiación son acumulativos. Se ha determinado como dosis umbral los 100 mGy. Los rayos X y la tomografía computarizada generan las dosis más altas, pero a menudo se pueden realizar de manera segura con la protección abdominal apropiada (12)
En cuanto al tratamiento del cáncer gástrico durante la gestación, aún no se dispone de estudios aleatorizados controlados, solo reportes de casos que describen efectos teratogénicos relacionados con el uso de radioterapia y agentes quimioterapéuticos, siendo estos documentados predominantemente en el primer trimestre de la gestación (9). La mayoría de agentes quimioterapeuticos son clasificados según la Food and Drug Administration (FDA) en la categoría D (13).

La cirugía rara vez está contraindicada; idealmente debe realizarse en el segundo trimestre. La técnica de la cirugia abdominal require especial atención dado que el utero en crecimiento puede desplazar los organos internos dependiendo la edad gestacional, se recomienda el abordaje mediante incisión mediana para lograr una mayor exposición. No necesariamente se debe terminar la gestación para iniciar el tratamiento (14). El 5 fluoracilo ha demostrado aumento de la supervivencia en el cáncer colorectal (10) aún no se dispone de estudios epidemiológicos en cuanto a anomalías congénitas en hijos de madres expuestas a dicho quimioterapéutico $(15,16)$ young age or female sex. Experimental and epidemiological data suggest a protective effect of oestrogen against the induction of (intestinal, solo series de casos que muestran abortos en alrededor del $50 \%$ de las pacientes en primer trimestre y restricción del crecimiento intrauterine (RCIU) en segundo y tercer trimester lo que hace importante la vigilancia del crecimiento fetal en las gestantes que cursan con cáncer que require manejo con quimioterapia (17-20)

\section{CASO CLÍNICO \# 1}

Presentamos el caso de una paciente de 34 años G4P3 con gestación de 18 semanas y 3 días al ingreso remitida de un primer nivel de atención por cuadro clínico de un mes de evolución caracterizado por dolor lumbosacro izquierdo de intensidad progresiva, con impresión diagnóstica de urolitiasis, evidencia de anemia normocítica normocrómica con $\mathrm{Hb} 7.5 \mathrm{~g} / \mathrm{dL}$ y ecografía renal y de vías urinarias institucional normal. La ecografía de abdomen total mostró hallazgos sugestivos de torsión ovárica izquierda sin abdomen quirúrgico en ese momento. Por hallazgo de anemización aguda en 24 horas con hemoglobina de control de $6.4 \mathrm{~g} / \mathrm{dL}$ y melenas, se realiza endoscopia de vías digestivas altas que muestra hallazgos macroscópicos compatibles con carcinoma gástrico Bormann III, realizandose dicho diagnóstico a las 19 semanas y 3 días de gestación, histopatología reporta adenocarcinoma gástrico difuso. 
Se realizan estudios de extensión con el fin de estadificar la enfermedad neoplásica, entre ellos Resonancia magnética que no muestra metástasis hepaticas ni pulmonares, evidencia adenopatías perigástricas y engrosamiento de la pared gástrica, adicionalmente, confirma hallazgo de masa ovárica izquierda de 10 x $10 \mathrm{~cm}$ y metástasis óseas a sacro y fémur proximal que contraindican parto vaginal (Figura 1).
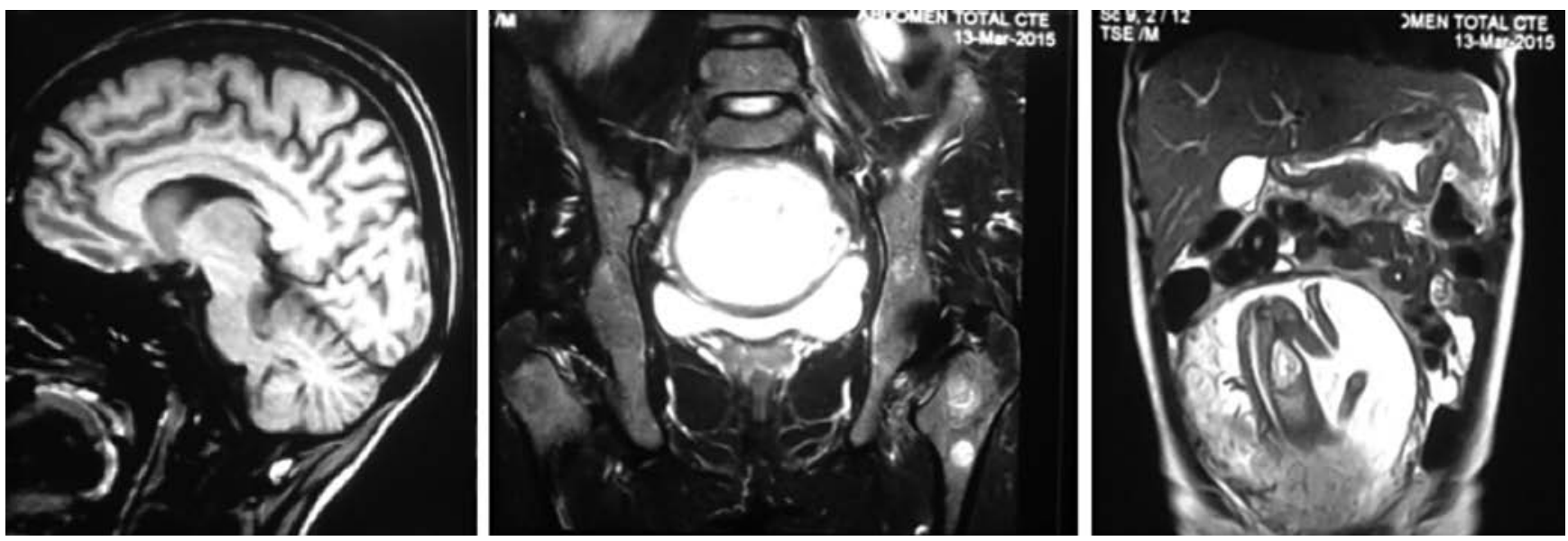

Figura 1. RMN cerebro, abdomen y pelvis. Izquierda: RMN cerebral, corte sagital sin evidencia de enfermedad metastásica. Centro: Metástasis a fémur izquierdo proximal. Derecha: Engrosamiento difuso de pared gástrica.

A las 20 semanas y 6 días cirugía general realiza laparotomía exploratoria con hallazgos quirúrgicos de tumor gástrico de $10 \times 15 \mathrm{~cm}$ que comprometía la curvatura mayor y menor con compromiso del hilio esplénico y del páncreas en sus tres porciones. La biopsia por congela- ción confirma carcinoma gástrico (Figura 2). Se realiza ooforectomía izquierda paliativa y biopsia de ganglios pericavales cuya patología confirmó carcinoma gástrico metastásico. No es posible realizar cirugía curativa por extensa carga tumoral.
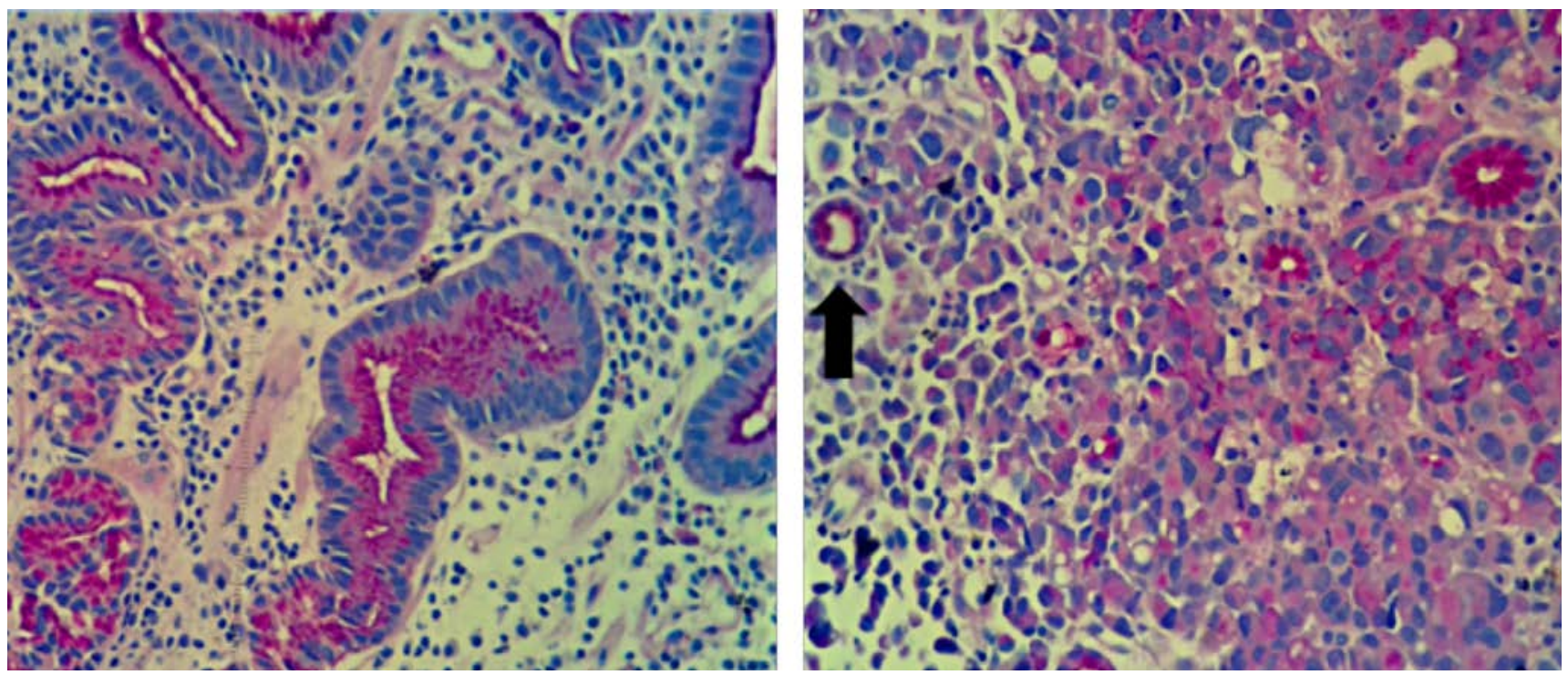

Figura 2. Descripción microscópica de carcinoma gástrico difuso. Izquierda: Tejido epitelial glandular gástrico con atipias Derecha. Tejido epitelial gástrico, la flecha indica célula en anillo de sello. 
Los hallazgos histopatológicos de la masa ovárica fueron compatibles con enfermedad metastásica y tumor de Krukenberg (Figura 3).
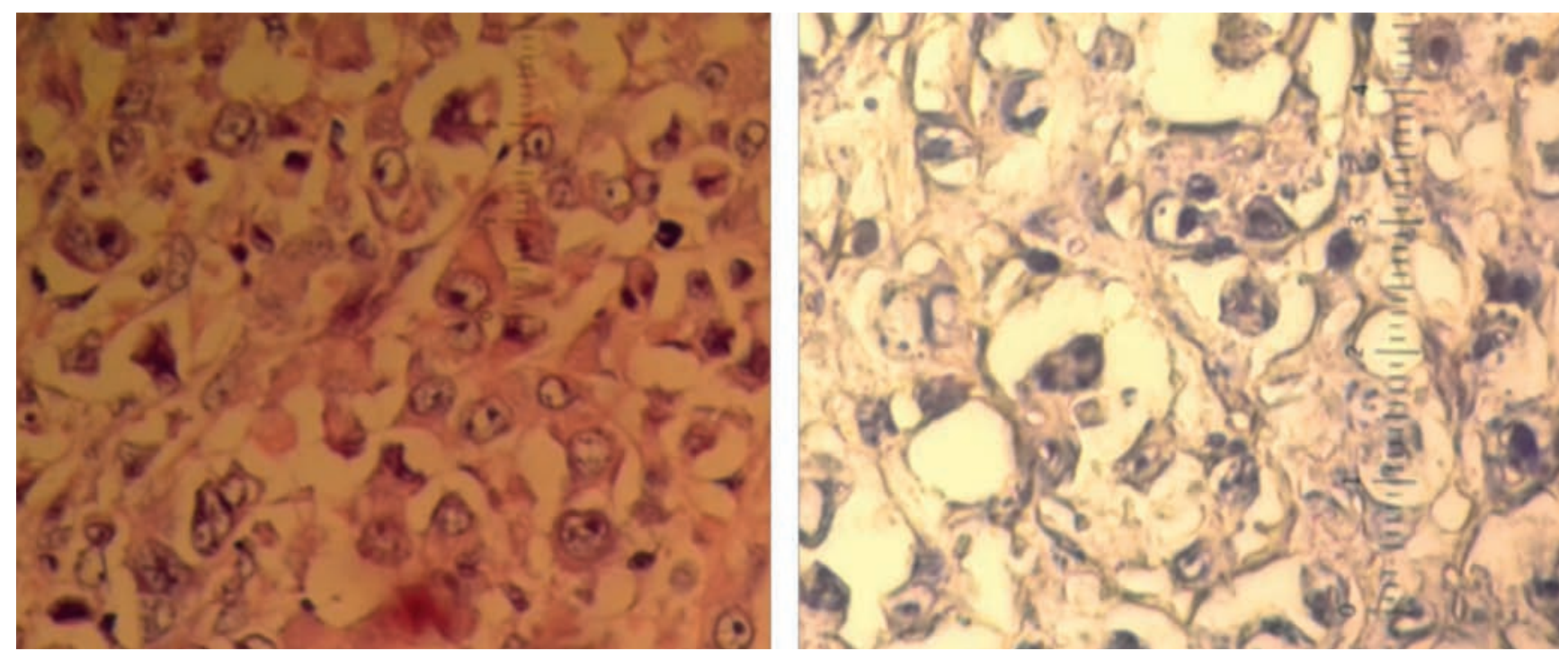

Figura 3. Descripción microscópica de biopsia de tejido ovárico. Izquierda: Células epiteliales con núcleos pleomórficos, nucleolo prominente, citoplasma claro a eosinófilo pobremente cohesivo. Mitosis atípicas. Derecha: Inmunohhistoquímica que favorece origen metastásico gástrico.

Debido al hallazgo de enfermedad neoplásica avanzada (estadío IV) y el deseo de la paciente de continuar con su embarazo, se determina en junta de medicina maternofetal y hematooncología realizar manejo paliativo con esquema quimioterapéutico FOLFIRI, compuesto por 5 fluoracilo, ácido folínico e irinotecán, los cuales han demostrado en las series de casos ser los menos lesivos sobre el bienestar fetal. Dicha decisión es llevada a comité de ética médica institucional y en común acuerdo con la paciente se decide continuar con la gestación.

A las 22 semanas y 6 días de gestación se inicia quimioterapia FOLFIRI completando 5 ciclos a la semana 32 con único hallazgo de placenta hipermadura a la semana 29 , este esquema no se continua por hallazgo de restricción del crecimiento intrauterino a la semana 33 que progresa a estadío III (21) dado por índice de pulsatilidad del ducto venoso mayor al percentil 95 con un peso fetal estimado de 1492 gr (menor a percentil 3) motivo por el cual la paciente es llevada a cesárea a las 33 semanas y 5 días después de recibir esquema completo de corticoides antenatales, obteniendo recién nacido vivo de sexo masculino con peso de 1582 gramos que requirió hospitalización durante un mes en cuidados básicos neonatales para ganancia ponderal y estudios de extensión los cuales fueron normales.
Durante el puerperio se realizó ablactación, se documenta enfermedad metastasica poliostótica con gamagrafía ósea, metastasis a mama derecha e izquierda mediante biopsia trucut y compromiso retroperitoneal con hidronefrosis secundaria izquierda. La paciente recibió 2 ciclos adicionales de quimioterapia intrahospitalaria la cual fue suspendida por múltiples episodios de hemorragia de vías digestivas altas y anemia severa que requieren transfusión de hemoderivados, cambio a radioterapia femoral y gástrica 10 sesiones y posteriormente quimioterapia con capecitabina. Se dió egreso para permitir alojamiento conjunto con el bebé en su domicilio y continuar seguimiento ambulatorio sin embargo la paciente fallece a los 2 meses en su domicilio por su enfermedad. No se realizó necropsia a solicitud de familiares y el seguimiento ambulatorio del recien nacido no ha mostrado efectos adversos de la quimioterapia.

\section{CASO CLINICO \#2}

Presentamos el caso de una paciente de 31 años G1P0 con gestación de 26 semanas y 6 días al ingreso quien consulta por cuadro clínico de 2 meses de evolución de emesis persistente, epigastralgia, distension abdominal, hiporexia y perdida de peso de $4 \mathrm{~kg}$ durante el 
embarazo. La pacientes al ingreso en estado de desnutrición con IMC 17.3, se hospitaliza para estudio mediante perfil hepático que evidencia aumento de las transaminasas con una AST en 155.6 y ALT en 173.7, electrolitos con hipokalemia (potasio en 2.65) e hiponatremia (sodio 130) las cuales se corrigen; se toma ecografía hepatobiliar que identifica engrosamiento en las paredes de la region antopilorica y endoscopia de vias digestivas altas que reporta una lesion tumoral de aspecto infiltrante con lesion dominante antro pilorica e incisura Bormann III y sindrome pilorico interrogado. El estudio histopatológico con biopsia confirma adenocarcinoma gástrico difuso sin invasión linfovascular, gastritis crónica moderada y Helicobacter Pylori positivo (FIGURA 4). La ecografía obstetrica del ingreso reporta Restricción del crecimiento intrauterino por lo que se continuo seguimiento con Doppler fetoplacentario. Se inicia manejo multidisciplinario en conjunto con los servicios de medicina maternofetal, psicología oncológica, cirugia oncológica, oncología clínica y soporte nutricional. Se inicia nutrición enteral mediante sonda avanzada a duodeno e inhibidores de bomba de protones. Debido a que el embarazo era planeado y deseado, la paciente en conjunto con su pareja decide continuar con la gestación.
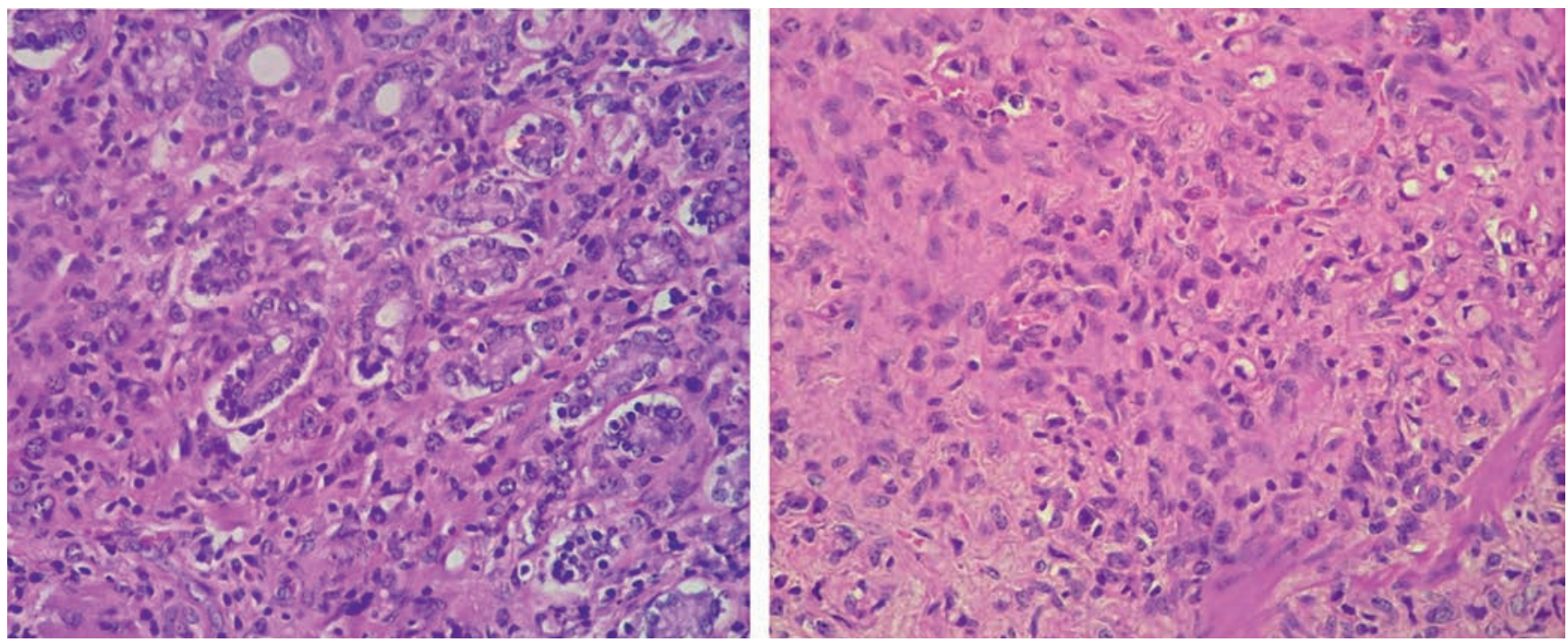

Figura 4. Descripción microscópica de adenocarcinoma gástrico difuso. Izquierda: Tejido epitelial glandular gástrico con atipias y cambios epiteliales reactivos asociados. Derecha. Tejido epitelial gástrico con células en anillo de sello.

Se realizan estudios de extensión para estadificar la enfermedad y definir manejo quirurgico mediante TAC Toracoabdominal que evidencia útero gestante con curso normal, tumor gástrico antropilórico que no penetra la pared aparentemente, sin infiltrar estructuras adyacentes, sin metastasis, sin evidencia de conglomerados celiacos, ni implantes epiploicos o peritoneales. (FIGURA 5). Con estos hallazgos se considera paciente candidata a gastrectomía posiblemente total con disección DII por parte de cirugía oncológica posterior a la terminación del embarazo. Se realiza junta multidisciplinaria en donde se decide desembarazar a las $34 \mathrm{se}$ manas por cesárea siempre y cuando se documentara bienestar fetal, previa maduración pulmonar fetal y en el puerperio llevar a procedimiento quirurgico propuesto por cirugía oncológica.
A las 34 semanas de gestación la paciente es llevada a cesárea, obteniendo un recién nacido vivo masculino de peso $1684 \mathrm{gr}$, talla $42.5 \mathrm{~cm}$ y apgar de 8/10 y 10/10 al minuto y 10 minutos respectivamente, que se hospitaliza en la unidad neonatal por bajo peso. La paciente presenta una adecuada evolución durante su puerperio y el dia 19 postparto es llevada a gastrectomía total con vaciamiento ganglionar del área celíaca, anastomosis esofagoduodenal y reconstrucción en Y de Roux. Dentro de los hallazgos intraoperatorios se encuentra un tumor de aproximadamente $10 \mathrm{~cm}$ de diámetro en el área antral prepilórica, sin compromiso de la serosa, adenopatías en área celíaca de 1 y $3 \mathrm{~cm}$ de diámetro, macroscópicamente no sugestivos de actividad tumoral. No se evidenció actividad tumoral a distancia, no metástasis hepáticas ni implantes peritoneales. La pa- 
tología confirma adenocarcinoma gástrico difuso grado 3 (mal diferenciado) con tamaño tumoral de 9x7x2 cm en region antropilórica con compromiso hasta la serosa con bordes de sección proximal y distal libres de tumor, invasión linfovascular presente e invasión perineural identificada, HER-2 Negativo, Ki 67 del 30\%, 5 ganglios de curvatura mayor y menor con compromiso tumoral, donas anastomóticas negativas para infiltración tumoral. La paciente presenta adecuada evolución en el postoperatorio, al quinto día se realiza estudio de vías digestivas altas para evaluar estado de anastomosis esofagoyeyunal encontrándola indemne y con adecuado paso del medio de contraste, sin extravasaciones, por lo que se inicia tolerancia a la vía oral con adecuada respuesta. Se retira nutrición enteral y se da egreso. La paciente continua en seguimiento por cirugía oncológica y hematooncología actualmente, tiene pendiente definir inicio de quimioterapia.
1

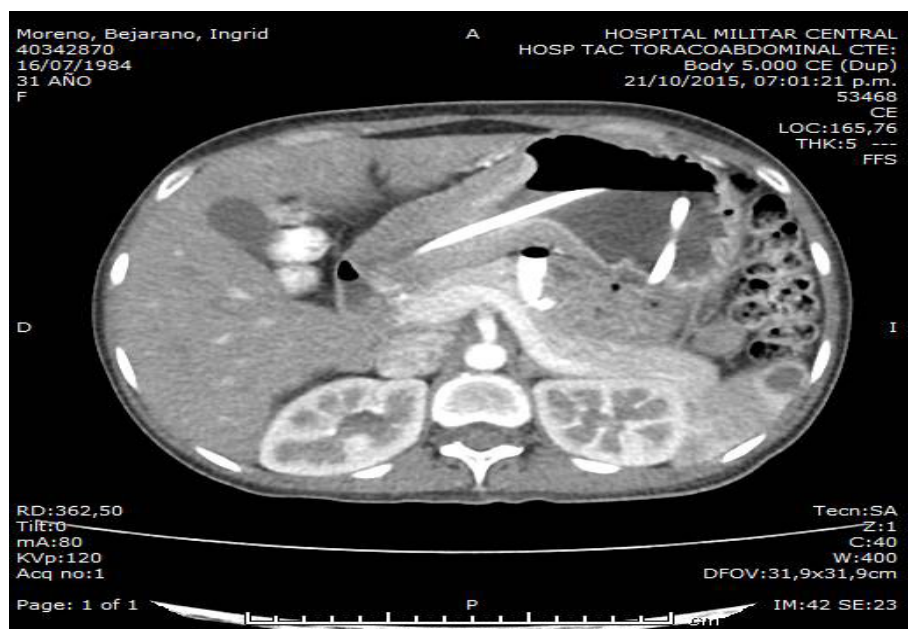

3

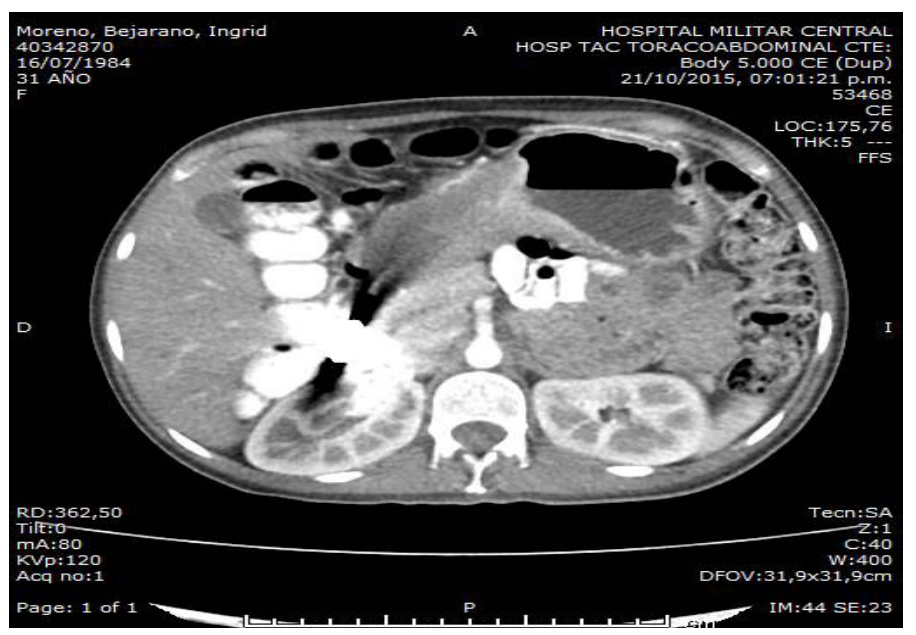

2

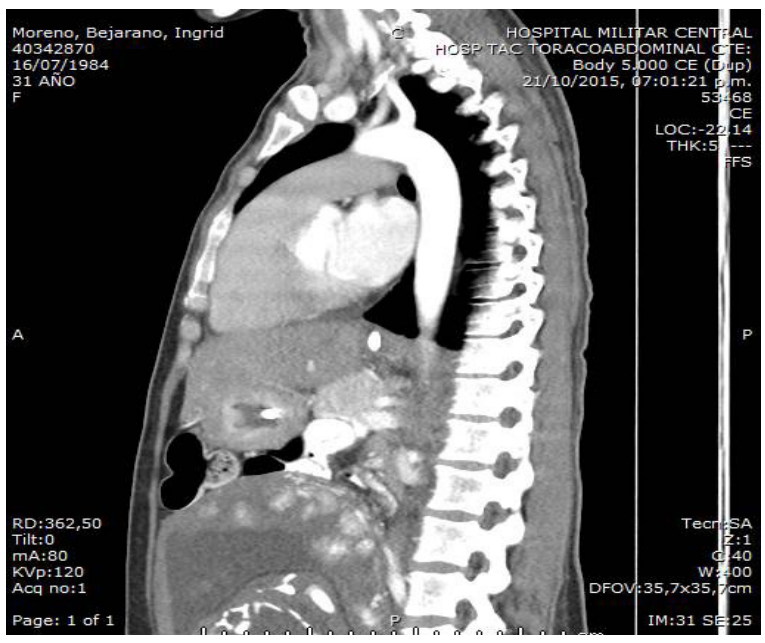

4

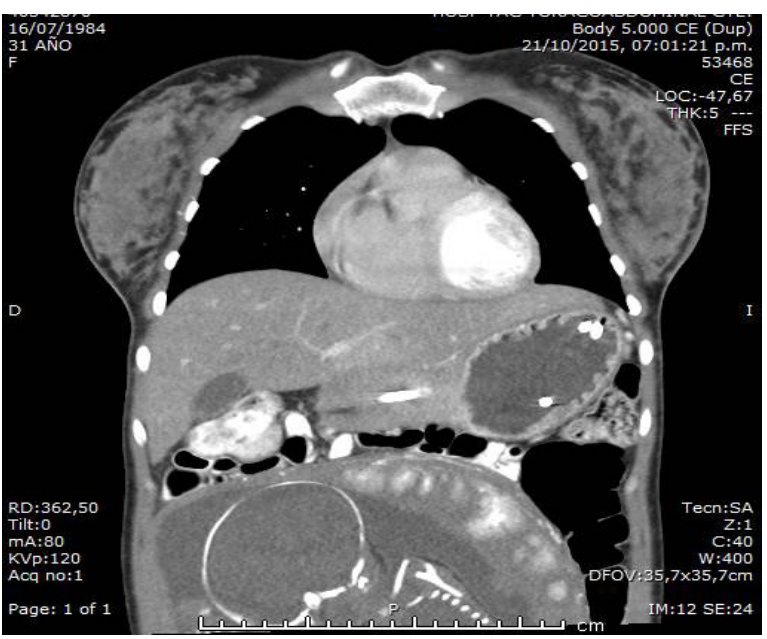

Figura 5. TAC Toracoabdominal. 1: Engrosamiento y perdida de pliegues en la región antropilórica. 2: engrosamiento de la mucosa gástrica a nivel de la curvatura mayor. 3: Engrosamiento en la región antropilórica. 4: engrosamiento de la mucosa Gástrica a nivel de la curvatura mayor. 


\section{Discusión}

La incidencia de cáncer gástrico en la población general varía de acuerdo a la localización geográfica, siendo mayor en los países en vías de desarrollo donde coexisten los factores de riesgo reconocidos para el desarrollo de la enfermedad: infección por H. Pilori y uso de preservantes de alimentos (11)325 consecutive patients, there were 152 young patients under 40 years of age (57 males and 95 females. Antes de 1980 el cáncer gástrico era la primera causa de mortalidad por cáncer a nivel mundial, lugar que posteriormente fué ocupado por el cáncer de pulmón (22)"ISSN" : "15424863 (Electronic debido a una reducción de su incidencia posiblemente por la identificación y tratamiento temprano de la infección por $\mathrm{H}$. pilori, sin embargo, se ha observado un incremento de su presentación en edades cada vez más tempranas (23).

Colombia se encuentra dentro de los países con mayor incidencia de cáncer gástrico a nivel mundial; según datos del GLOBOCAN de la OMS del año 2012, en nuestro país dicha entidad sigue ocupando el primer lugar dentro del top 5 de cánceres en incidencia y mortalidad, con una incidencia en la población femenina de 8.97 casos por cada 100.000 habitantes y de 18.89 casos por cada 100.000 habitantes en población masculina, sin embargo, esta patología afecta solo el 3.5 al $6.5 \%$ de los pacientes menores de 40 años siendo en este grupo más frecuente en el sexo femenino (11)325 consecutive patients, there were 152 young patients under 40 years of age $(57$ males and 95 females. En pacientes gestantes su incidencia oscila alrededor 0.016 $\%$ (1) a $0.1 \%(24,25)$ a total of 3,256 patients with gastric cancer were admitted to our institute. Among these patients, $3(0.1 \%$.

La mayoría de pacientes con cáncer gástrico son asintomáticos manifestando signos y síntomas de la enfermedad cuando esta ya se encuentra en estadios avanzados.

El diagnóstico de cáncer en la gestación es una situación potencialmente devastadora tanto para la gestante como para su familia (26). Solo el $25 \%$ de los pacientes con diagnóstico de carcinoma gástrico son candidatos a resección potencialmente curativa, de este último grupo la gran mayoría fueron diagnosticados mediante programas de tamizaje que en nuestro medio no se realizan rutinariamente en contraste con otros países de alta prevalencia de enfermedad como lo son Japón, Venezuela y Chile (27).
Según la revision de la literatura elaborada por Jaspers en 1999 (15) sólo se habían reportado 131 casos de cáncer gástrico en el embarazo en las últimas tres décadas, de los casos revisados encontró que más del 95\% de los canceres gástricos en gestantes fuera de Japón eran mal diferenciados y generalmente de tipo difuso como el de nuestras pacientes.

En términos de sobrevida, esta es comparable con la población no gestante y al parecer el embarazo, la edad materna y el sexo no empeoran la supervivencia, se han reportado tasas de supervivencia a 3 años del $8 \%$ con más del $70 \%$ de recién nacidos sobrevivientes. En la literatura no existen reportes de metástasis al feto $(15,28)$.

La cirugía constituye un pilar importante en el tratamiento del cáncer gástrico, desempeñando el mismo papel en la gestación, siendo curativa en el 50-60\% de los casos diagnosticados en estadíos tempranos como en el Segundo caso $(29,30)$. Preferentemente se debe realizar en segundo trimestre, cuando la organogénesis se encuentra completa y el tamaño del útero no interfiere con el procedimiento.

Se realizan estudios de extensión con el fin de estadificar la enfermedad neoplásica, entre ellos Resonancia magnética que no muestra metástasis hepaticas ni pulmonares, evidencia adenopatías perigástricas y engrosamiento de la pared gástrica, adicionalmente, confirma hallazgo de masa ovárica izquierda de $10 \mathrm{x}$ $10 \mathrm{~cm}$ y metástasis óseas a sacro y fémur proximal que contraindican parto vaginal (Figura 1).

A las 20 semanas y 6 días cirugía general realiza laparotomía exploratoria con hallazgos quirúrgicos de tumor gástrico de 10 x $15 \mathrm{~cm}$ que comprometía la curvatura mayor y menor con compromiso del hilio esplénico y del páncreas en sus tres porciones. La biopsia por congelación confirma carcinoma gástrico (Figura 2).

Se realiza ooforectomía izquierda paliativa y biopsia de ganglios pericavales cuya patología confirmó carcinoma gástrico metastásico. No es posible realizar cirugía curativa por extensa carga tumoral.

Los hallazgos histopatológicos de la masa ovárica fueron compatibles con enfermedad metastásica y tumor de Krukenberg (Figura 3). 


\section{Conclusiones}

- El diagnóstico de cáncer durante la gestación no implica siempre la interrupción de la misma.

- Se debe individualizar cada caso teniendo en cuenta el estadío de la enfermedad y la desición materna de continuar ó no con la gestación

- Aún no existen estudios clínicos estadísticamente significativos que establezcan una guía de manejo en cuanto al tratamiento del cáncer gástrico en la gestación se refiere.

- Los efectos adversos de la quimioterapia en la gestación reportados hasta el momento en la literatura son escasos y en estudios animales, lo reportado hasta ahora incluye anomalías como RCIU y parto pretérmino sin embargo en la mayoría de los casos son recién nacidos sanos si esta es administrada después del período de la organogénesis.

- El cáncer gástrico en gestantes suele diagnosticarse aún más tardíamente que en la población general por la similitud de su presentación clínica y los síntomas propios del embarazo, lo cual lleva a un peor pronóstico con graves consecuencias tanto maternas como fetales.

\section{Conflicto de intereses: Ninguno}

\section{Agradecimientos}

Agradecemos al servicio de Oncología del Hospital Militar Central por el acompañamiento y la labor llevada a cabo en el tratamiento de las pacientes de los casos presentados, así mismo agradecemos al servicio de patología por permitirnos el acceso a las placas histológicas de las pacientes.

\section{Referencias}

1. Nakagawa K. Four cases of gastric cancer during pregnancy. J Jpn Coll Surg. 2000;25:892-5.

2. Kurabayashi T, Isii K, Suzuki M, Takakuwa K, Shibazaki Y, Ozawa T, et al. Advanced gastric cancer and a concomitant pregnancy associated with disseminated intravascular coagulation. Am J Perinatol. United States; 2004 Jul;21(5):295-8.

3. Samarasinghe A, Shafi MI. Cancer in pregnancy. Obstet Gynaecol Reprod Med [Internet]. 2014;24(11):333-9.

Available from: http://www.sciencedirect.com/science/article/pii/S175172141400178X

4. Ueo H, Matsuoka H, Tamura S, Sato K, Tsunematsu Y,
Kato T. Prognosis in gastric cancer associated with pregnancy. World J Surg. United States; 1991;15(2):293-7, discussion 298.

5. Dunkelberg JC, Barakat J, Deutsch J. Gastrointestinal, pancreatic, and hepatic cancer during pregnancy. Obstet Gynecol Clin North Am. United States; 2005 Dec;32(4):641-60.

6. Morice P, Uzan C, Gouy S, Verschraegen C, Haie-Meder C. Gynaecological cancers in pregnancy. Lancet (London, England). England; 2012 Feb;379(9815):558-69.

7. Saif MW. Management of colorectal cancer in pregnancy: a multimodality approach. Clin Colorectal Cancer. United States; 2005 Nov; 5(4):247-56.

8. Krishnansu S. Tewari. Cancer in Pregnancy. In: Clinical Gynecologic Oncology. Seventh. 2007. p. 467-531.

9. Voulgaris E, Pentheroudakis G, Pavlidis N. Cancer and pregnancy: a comprehensive review. Surg Oncol. Netherlands; 2011 Dec;20(4):e175-85.

10. Martin MJ. Current stage-specific chemotherapeutic options in colon cancer. Expert Rev Anticancer Ther. England; 2005 Aug;5(4):695-704.

11. Maeta M, Yamashiro H, Oka A, Tsujitani S, Ikeguchi M, Kaibara N. Gastric cancer in the young, with special reference to 14 pregnancy-associated cases: analysis based on 2,325 consecutive cases of gastric cancer. J Surg Oncol. United States; 1995 Mar;58(3):191-5.

12. Needleman S, Powell M. Radiation hazards in pregnancy and methods of prevention. Best Pract Res Clin Obstet Gynaecol. Netherlands; 2016 May;33:108-16.

13. Lambertini M, Peccatori FA, Azim HAJ. Targeted agents for cancer treatment during pregnancy. Cancer Treat Rev. Netherlands; 2015 Apr;41(4):301-9.

14. Moran BJ, Yano H, Al Zahir N, Farquharson M. Conflicting priorities in surgical intervention for cancer in pregnancy. Lancet Oncol. England; 2007 Jun;8(6):536-44.

15. Jaspers VK, Gillessen A, Quakernack K. Gastric cancer in pregnancy: do pregnancy, age or female sex alter the prognosis? Case reports and review. Eur J Obstet Gynecol Reprod Biol. Ireland; 1999 Nov;87(1):13-22.

16. Dupont JBJ, Lee JR, Burton GR, Cohn IJ. Adenocarcinoma of the stomach: review of 1,497 cases. Cancer. United States; 1978 Mar;41(3):941-7.

17. Duarte I, Llanos O. Patterns of metastases in intestinal and diffuse types of carcinoma of the stomach. Hum Pathol. United States; 1981 Mar;12(3):237-42.

18. Kim S-H, Abd Halim SR, Siddiqui N, Park W-HE. Disseminated cancer in pregnancy: krukenberg tumour. Case Rep Obstet Gynecol. United States; 2014;2014:216969.

19. DAGG CP. Sensitive stages for the production of developmental abnormalities in mice with 5-fluorouracil. Am J Anat. United States; 1960 Mar;106:89-96.

20. Furukawa H, Iwanaga T, Hiratsuka M, Imaoka S, Ishikawa $\mathrm{O}$, Kabuto T, et al. Gastric cancer in young adults: growth accelerating effect of pregnancy and delivery. J Surg Oncol. United States; 1994 Jan;55(1):3-6.

21. Figueras F, Gratacos E. Update on the diagnosis and classification of fetal growth restriction and proposal of a sta- 
ge-based management protocol. Fetal Diagn Ther. Switzerland; 2014;36(2):86-98.

22. Jemal A, Bray F, Center MM, Ferlay J, Ward E, Forman D. Global cancer statistics. CA Cancer J Clin. United States; 2011;61(2):69-90.

23. Correa P. Gastric cancer: two epidemics? Vol. 56, Digestive diseases and sciences. United States; 2011. p. 1585-6; author reply 1586.

24. Kodama I, Takeda J, Koufuji K, Yano S, Hanzawa M, Shirouzu K. Gastric cancer during pregnancy. Kurume Med J. Japan; 1997;44(3):179-83.

25. Salani R, Billingsley CC, Crafton SM. Cancer and pregnancy: an overview for obstetricians and gynecologists. Am J Obstet Gynecol. United States; 2014 Jul;211(1):714.

26. Parangi S, Levine D, Henry A, Isakovich N, Pories S. Surgical gastrointestinal disorders during pregnancy. Am J Surg [Internet]. 2007;193(2):223-32. Available from: http://www.sciencedirect.com/science/article/pii/ S0002961006007604

27. Ministry of Health L and W, JAPAN. Vital Statistics [Internet]. Available from: http://www.mhlw.go.jp/english/ database/db-hw/index.html

28. Al-Ibrahim A, Parrish J, Dunn E, Swallow C, Maxwell C. Pregnancy and maternal outcomes in women with prior or current gastrointestinal malignancies. J Obstet Gynaecol Can. Netherlands; 2014 Jan;36(1):34-41.

29. Wilson JG. Use of rhesus monkeys in teratological studies. Fed Proc. United States; 1971;30(1):104-9.

30. Dotters-Katz S, McNeil M, Limmer J, Kuller J. Cancer and pregnancy: the clinician's perspective. Obstet Gynecol
Surv. United States; 2014 May;69(5):277-86.

31. Wilson JG. Abnormalities of intrauterine development in non-human primates. Acta Endocrinol Suppl (Copenh). Denmark; 1972;166:261-92.

32. Dreicer R, Love RR. High total dose 5-fluorouracil treatment during pregnancy. Wis Med J. United States; 1991 Oct;90(10):582-3.

33. Stadler HE, Knowles J. Fluorouracil in pregnancy: effect on the neonate. JAMA. United States; 1971 Jul;217(2):2145.

34. Amant F, Han SN, Gziri MM, Vandenbroucke T, Verheecke M, Van Calsteren K. Management of cancer in pregnancy. Best Pract Res Clin Obstet Gynaecol. Netherlands; 2015 Jul;29(5):741-53.

35. Lordick F, Siewert JR. Recent advances in multimodal treatment for gastric cancer: a review. Gastric Cancer. Japan; 2005;8(2):78-85.

36. Moehler M, Schimanski CC, Gockel I, Junginger T, Galle PR. (Neo)adjuvant strategies of advanced gastric carcinoma: time for a change? Dig Dis. Switzerland; 2004;22(4):34550 .

37. Ilancheran A, Low J, Ng JS. Gynaecological cancer in pregnancy. Best Pract Res Clin Obstet Gynaecol. Netherlands; 2012 Jun;26(3):371-7.

38. Aviles A, Neri N. Hematological malignancies and pregnancy: a final report of 84 children who received chemotherapy in utero. Clin Lymphoma. United States; 2001 Dec;2(3):173-7.

39. Backes $\mathrm{CH}$, Moorehead PA, Nelin LD. Cancer in pregnancy: fetal and neonatal outcomes. Clin Obstet Gynecol. United States; 2011 Dec;54(4):574-90. 